\title{
Incidence and Risk Factors for Liver Abscess After Thermal Ablation of Liver Neoplasm
}

\author{
Xiu-Feng Su, ${ }^{1,2} \mathrm{Na} \mathrm{Li},{ }^{3}$ Xu-Fang Chen, ${ }^{2}$ Lei Zhang, ${ }^{4}$ and Ming Yan $^{1,}{ }^{*}$ \\ ${ }^{1}$ Department of Geriatric Gastroenterology, Qilu Hospital, Shandong University, Jinan, China \\ ${ }^{2}$ Department of Oncology, Yantai Affiliated Hospital, Binzhou Medical University, Yantai, China \\ ${ }^{3}$ Department of Rheumatology, Yantai Affiliated Hospital, Binzhou Medical University, Yantai, China \\ ${ }^{4}$ Department of Infectious Diseases, Yantai Affiliated Hospital, Binzhou Medical University, Yantai, China \\ "Corresponding author: Ming Yan, Department of Geriatric Gastroenterology, Qilu Hospital, Shandong University, Jinan, China. E-mail: ymylh@163.com
}

Received 2015 November 11; Revised 2016 April 16; Accepted 2016 April 21.

\begin{abstract}
Background: Radiofrequency ablation (RFA) and microwave ablation (MWA) are the most frequently used thermal ablation methods for the treatment of liver cancer. Liver abscess is a common and severe complication of thermal ablation treatment.

Objectives: The objective of this study was to determine the incidence and risk factors of liver abscess formation after thermal ablation of liver cancer.

Materials and Methods: The clinical data of 423 patients who underwent 691 thermal ablation procedures for liver cancer were collected in order to retrospectively analyze the basic characteristics, incidence, and risk factors associated with liver abscess formation. Patients with multiple risk factors for liver abscess formation were enrolled in a risk factor group, and patients with no risk factors were enrolled in a control group. The chi-square test and multiple logistic regression analysis were used to analyze the relationship between the occurrence of liver abscesses and potential risk factors.

Results: Two hundred and eight patients underwent 385 RFA procedures, and 185 patients underwent 306 MWA procedures. The total incidence of liver abscesses was $1.7 \%$, while the rates in the RFA group (1.8\%) and MWA groups (1.6\%) were similar (P>0.05). The rates of liver abscesses in patients who had child-pugh class $B$ and class $C$ cirrhosis $(P=0.0486)$, biliary tract disease $(P=0.0305)$, diabetes mellitus $(\mathrm{P}=0.0344)$, and porta hepatis tumors $(\mathrm{P}=0.0123)$ were $4.0 \%, 6.7 \%, 6.5 \%$, and $13.0 \%$, respectively. There was a statistically significant difference between these four groups and the control group (all $\mathrm{P}<0.05$ ). The incidence of liver abscesses in the combined ablation and percutaneous ethanol injection $(\mathrm{PEI})$ group $(\mathrm{P}=0.0026)$ was significantly lower than that of the ablation group $(\mathrm{P}<0.05)$.

Conclusions: The incidence of liver abscesses after liver cancer thermal ablation is low. Child-Pugh Class B and Class C cirrhosis, biliary tract disease, diabetes mellitus, and porta hepatis tumors are four significant risk factors. Combined ablation and PEI reduces the rate of liver abscesses.
\end{abstract}

Keywords: Liver Neoplasm, Ablation, Liver Abscess, Incidence, Risk Factors

\section{Background}

Liver cancer is one of the most common malignancies in the world. At present, liver transplantation and surgical resection are still the most important treatments for liver cancer, but only a small number of patients receive these treatments in time. Image-guided tumor ablation plays an important role in the treatment of primary and metastatic liver cancer $(1,2)$. Radiofrequency ablation (RFA) and microwave ablation (MWA) are the most common local thermal ablation methods, and have been widely used in the treatment of liver cancer all over the world $(1,2)$. The effectiveness of thermal ablation methods for small liver cancers is equivalent to that of surgical resection (3-5). Thermal ablation is considered the frontline choice for the treatment of liver cancers, especially those that cannot be treated with surgical resection (6).
Although RFA and MWA are considered relatively safe and minimally invasive techniques, they can cause severe complications, such as intra-abdominal hemorrhage, liver abscess, biliary fistula, intestinal perforation, seeding, and skin burn; of these, liver abscess is one of the most common complications $(7,8)$. A good understanding of the complications that may occur post-treatment is the key to successful thermal ablation. Many studies have analyzed complications after RFA or MWA for liver cancer; however, most of these reports addressed clinical issues and treatment for liver abscesses following RFA. Few have undertaken statistical analyses of risk factors $(9,10)$. Although some studies have assessed risk factors for liver abscess formation after RFA, there has been little similar research on $\operatorname{MWA}(11,12)$. 


\section{Objectives}

The aim of this study was to retrospectively analyze the clinical records of 423 patients with liver cancer who underwent RFA or MWA, in order to determine the incidence of liver abscesses and to identify the risk factors for liver abscess formation.

\section{Materials and Methods}

\subsection{Patient Selection}

From July 2006 to July 2014, 423 patients with 767 liver tumors underwent a total of 691 thermal ablation therapy procedures at Qilu hospital of Shandong University and Weihai Municipal hospital. This retrospective study was approved by the ethics committee of Qilu hospital, allowing the investigators to review existing patient medical records. Because the study was retrospective, informed consent was waived. Informed consent was obtained for the original treatments of all patients. Both of the procedures (RFA and MWA) were explained to the patients, and the decision about which procedure to perform was based on the patient's economic condition, tumor size, and personal choice.

The inclusion criteria for the thermal ablation patients were hepatocellular carcinoma, intrahepatic cholangiocarcinoma or metastatic hepatic carcinoma, child-pugh class A or class B cirrhosis, or class $\mathrm{C}$ that transitioned to class B after treatment.

The exclusion criteria were an age of $>80$ years; an ASA score of grade IV or V, stable child-pugh class C cirrhosis, portal vein tumor thrombus or extrahepatic metastasis, platelet count of $<50 \times 10^{9} / \mathrm{L}$, liver atrophy with a tumor that was too large and exceeded the normal liver size, esophagogastric variceal hemorrhage within the previous one month, tumor dissemination, bile duct or hepatic venous cancer embolus, severe functional disorders or major organs, disturbance of consciousness or cachexia, refractory ascites, coagulation disorders or severe blood diseases that could not be treated, and HIV or immunosuppression (12).

\subsection{Thermal Ablation Procedure}

\subsubsection{Instruments and Equipment}

Thermal ablations were performed using two cooltip microwave treatment systems (Model MTC-3, Nanjing Forsea Microwave and Electron Institution, China; Model ECO-100A, Nanjing Yigao Company, China), and a cooltip radiofrequency ablation instrument (Model No. CTRF220, Tyco Valleylab) according to a previously described method (13). The ablations were performed under realtime ultrasound guidance with a $3.5-\mathrm{MHz}$ sector probe
(TOSHIBA, Xario Prime Ultrasound, SSA-6000). Philips Brilliance 64 multi-slice spiral CT, Siemens second-generation double-source SOMATOM Definition Flash CT, and Siemens 1.5T and 3.0T magnetic resonance imaging (Magnetom Trio A Tim) were used to evaluate the procedure in each case.

\subsubsection{Thermal Ablation Procedure}

All patients were treated under intravenous conscious sedation or local anesthesia. The procedures were performed by radiation intervention experts or surgical experts under strict sterile conditions in a dedicated interventional procedure room or operating room. The operators had extensive expertise and used real-time ultrasound, CT, or laparoscopy. Some procedures were performed via open abdominal surgery $(14,15)$. Briefly, the cool-tip microwave antenna or radiofrequency electrode was punctured percutaneously or directly into the center of each tumor $(16,17)$. During the ultrasound-guided procedure, there was no appropriate way of inserting into tumors close to diaphragmatic muscle, blocked by lung tissue. Therefore, it was necessary to inject $200-1,500 \mathrm{~mL}$ of physiological saline under the diaphragm to separate the tumors from the lung tissue. This method was also used to reduce organ injury when tumors were close to the gallbladder, heart, colon, stomach, or other organs $(18,19)$. A 21gauge percutaneous ethanol injection (PEI) needle was inserted into the liver tumor through the same attachment hole, beside the echo probe, before the thermal ablation in some patients. Pure ethanol (99.8\%) was then slowly injected into the tumor (20). In order to prevent tumor seeding, hemorrhage, and biloma, ablation was also conducted within the intrahepatic needle passage before withdrawal.

\subsubsection{Evaluation of Ablation Effects}

The ablation treatment was considered to be complete when the ablation zone (hyperechogenicity on ultrasound or a low-density region on CT) covered the whole tumor, and the ablative margin exceeded the edge of the tumor by $0.5-1 \mathrm{~cm}$ in three dimensions $(1,2,6,7)$. The effect of RFA or MWA was assessed with contrast-enhanced CT or MRI one month after the treatment. A reduction in alphafetoprotein levels also facilitated the assessment of effectiveness. If a remnant tumor or a new tumor was found on the CT or MR examination, supplementary treatments were performed.

\subsubsection{Diagnosis of Liver Abscess}

The criteria for the diagnosis of liver abscesses after thermal treatment were as follows: 1) a fever of $>38.5^{\circ} \mathrm{C}$ for more than 3 days, 2) a peripheral blood leukocyte count of $>10 \times 10^{9} / \mathrm{L}$ or $\left.<4 \times 10^{9} / \mathrm{L}, 3\right)$ repeated chills and fever, 
4) positive blood cultures or bacterial cultures by aspiration, and 5) septic shock. Peripheral enhancement of the ablation zone using $\mathrm{CT}$ and images showing pneumatosis in the ablation zone were considered diagnostic of a liver abscess if the patient met any of these five conditions.

\subsubsection{Follow-Up}

Follow-up visits for all recorded patients were completed. The first post-ablation follow-up visit occurred after the first complaint of symptoms. The total time of followup was six months. Baseline clinical data were recorded, including a detailed evaluation of the ablation treatment, the presence of liver abscesses, the amount of time taken for the abscesses to develop, culture results, treatment methods, and outcomes.

\subsection{Statistical Analysis}

We analyzed the incidence of liver abscesses after 691 thermal ablation procedures performed on 423 hepatic malignancy patients. We enrolled patients with multiple risk factors for liver abscess formation (21-23) into a risk factor group, and patients with no such risk factors into a control group. The data were presented in numbers (n), and the measurement data were represented by mean \pm SD. The statistical methods of the chi-square $\left(\chi^{2}\right)$ test and Fisher's exact probability test were used to compare the rates of liver abscesses between the different treatment methods.

The relationship between risk variables and liver abscess formation was analyzed with a multiple logistic regression analysis. A P value of $<0.05$ was considered to indicate statistical significance. The statistical analyses were performed with SPSS for Windows version 13.0 (SPSS Inc., Chicago, IL, USA).

\section{Results}

\subsection{Baseline Clinical Characteristics of the Patients (Table 1)}

This study's patients included 338 males and 85 females between the ages of 23 and 79 years. Of the 423 patients analyzed, 172 were diagnosed by percutaneous biopsy; of these, 108 had hepatocellular carcinoma and 64 had hepatic metastasis. Two hundred and fifty-nine patients were diagnosed with typical imaging examinations and the presence of tumor markers. The median diameter of the tumors was $3.2 \pm 2.3 \mathrm{~cm}$ (range $0.5-8.5 \mathrm{~cm}$ ). With regard to tumor position, there were 332 tumors in the left lobe of the liver and 435 tumors in the right lobe. Fortysix patients had tumors in the porta hepatis region. Two hundred and ninety patients had solitary tumors, and 133
Table 1. Clinical Characteristics of Patients and Tumors ${ }^{\mathrm{a}}$

\begin{tabular}{|c|c|}
\hline Patient and Tumor Characteristics & Value \\
\hline Age (23 - 79) & $57.0 \pm 14.8$ \\
\hline \multicolumn{2}{|l|}{ Gender } \\
\hline Male/female & $338 / 85$ \\
\hline \multicolumn{2}{|l|}{ Type of tumor } \\
\hline HCC/MHC/IHCC & $352 / 64 / 7$ \\
\hline Quantity of tumors & 767 \\
\hline Tumor diameter (0.5 - 8.5), cm & $3.5 \pm 2.3$ \\
\hline \multicolumn{2}{|l|}{ Tumor position (stage) } \\
\hline $\mathrm{I} / \mathrm{II} / \mathrm{III} / \mathrm{IV}$ & $3 / 143 / 151 / 138$ \\
\hline V/VI/VII/VIII & $88 / 152 / 43 / 48$ \\
\hline First porta hepatic tumor & 46 \\
\hline \multicolumn{2}{|l|}{ Quantity of tumors } \\
\hline Solitary/multiple & 290/133 \\
\hline \multicolumn{2}{|l|}{ Child-Pugh classification } \\
\hline $\mathrm{A} / \mathrm{B} / \mathrm{C}$ & $268 / 138 / 17$ \\
\hline Liver cirrhosis & 282 \\
\hline Alcoholism history & 32 \\
\hline Biliary tract disease & 53 \\
\hline Cholangiocarcinoma & 7 \\
\hline Diabetes mellitus & 56 \\
\hline Ever underwent TACE & 205 \\
\hline \multicolumn{2}{|l|}{ Preoperative blood biochemical indexes } \\
\hline ALT, U/L & $43.4 \pm 2.8$ \\
\hline Serum albumin, $\mathbf{g} / \mathbf{L}$ & $37.8 \pm 3.2$ \\
\hline Total bilirubin, $\mu \mathrm{mol} / \mathbf{L}$ & $27.2 \pm 7.4$ \\
\hline Preoperative antibiotic treatment & 106 \\
\hline \multicolumn{2}{|l|}{ Anesthesia } \\
\hline Local/intravenous & $260 / 163$ \\
\hline \multicolumn{2}{|l|}{ Puncture method } \\
\hline Percutaneous/abdominal/laparoscopic & $407 / 13 / 3$ \\
\hline \multicolumn{2}{|l|}{ Thermal ablation process } \\
\hline RFA/MWA & $385 / 306$ \\
\hline Thermal ablation time, min & $16.2 \pm 7.5$ \\
\hline \multicolumn{2}{|l|}{ Diagnosis method of liver abscess } \\
\hline $\mathrm{CT} / \mathrm{MR} /$ puncture fluid culture & $9 / 2 / 1$ \\
\hline
\end{tabular}

${ }^{\mathrm{a}}$ Values are expressed as No. or mean $\pm \mathrm{SD}$.

had multiple tumors. The numbers of patients with childpugh class A, class B, and class C cirrhosis were 268, 138, and 17 , respectively. Some class $C$ patients became class B 
after drug treatment. Among the 423 patients, the numbers with liver cirrhosis, alcoholism, biliary tract disease, and diabetes mellitus were 282, 32, 53 and 56, respectively. A total of 205 patients underwent transcatheter arterial chemoembolization (TACE) 15 to 388 days before accepting ablation treatment. The biliary tract conditions associated with the patients included intrahepatic cholangiocarcinoma, bile duct stones, biliary stent placement, bilioenteric anastomosis, and endoscopic sphincterotomy (EST). The mean blood ALT value of the 423 patients was $43.4 \pm$ $2.8 \mathrm{U} / \mathrm{L}$, mean albumin was $37.8 \pm 3.2 \mathrm{~g} / \mathrm{L}$, and mean total bilirubin was $27.2 \pm 7.4 \mu \mathrm{mol} / \mathrm{L}$. Before the operation, 106 patients had prophylactic antibiotic treatment. Local anesthesia was used in 260 patients and intravenous anesthesia was used in 163 . The three puncture methods were percutaneous $(n=407)$, open abdominal $(n=13)$, and laparoscopy-assisted $(\mathrm{n}=3)$. During the study period, 238 patients underwent 385 RFA procedures, and 185 patients underwent 306 MWA procedures. The mean thermal ablation time was $13.2 \pm 7.3$ minutes. The 12 patients with liver abscesses were diagnosed with contrast-enhanced CT $(\mathrm{n}=$ $9)$, contrast-enhanced MRI $(n=2)$, or cultures $(n=1)$.

\subsection{Clinical Characteristics of Liver Abscess Patients (Table 2 and Figures 1 and 2)}

Fifteen liver abscesses developed in 12 patients. These were identified using CT or MRI, except for one patient who had bacteria cultured from aspirated fluid in the ablation zone. Ten patients had fever and epigastric pain 3 - 28 days after the operation, and the other two had chills and tachycardia. For nine of these 12 patients, the ablation region and surrounding areas showed pneumatosis on CT images (Figure 1B and 2B). Blood or aspiration cultures from seven of the 12 patients were positive. Seven of the 12 patients had been treated with intravenous or oral antibiotics before the operation. Four of the 15 abscesses were punctured following sonographic guidance for drainage. Seven abscesses were drained with 10 - 14F catheters (Figure 2D) using sonographic guidance. All catheters were removed 10 - 50 days later, with no long-lasting sequelae. For some patients, drainage of multiple abscesses was performed (Figure 1C). Two patients were treated only with antibiotics, while one patient needed surgical treatment after percutaneous drainage had failed. Eleven of the patients with liver abscesses recovered within 10 - 60 days, but the remaining one died due to septic shock.

\subsection{Analysis of Incidence and RiskFactors of Liver Abscesses (Ta- bles 3 and 4)}

Among the 385 RFA procedures, liver abscesses developed in seven cases (1.9\%), while among the 306 MWA procedures, liver abscesses developed in five cases (1.6\%) ( $\mathrm{P}=$
0.8212). There were no liver abscesses in the 73 patients who underwent thermal ablation combined with PEI. This was significantly lower than in the non-combined treatment group (1.9\%) $(\mathrm{P}=0.014)$. Liver abscesses occurred in nine (1.6\%) of the 569 procedures performed on male patients, and in three $(2.4 \%)$ of the 122 procedures performed on female patients. Of the 301 procedures on patients with left lobe tumors, five (1.6\%) developed liver abscesses. Of the 390 procedures on patients with right lobe tumors, seven (1.8\%) developed liver abscesses. In the liver abscess group, the mean tumor diameter was $4.2 \pm 2.6 \mathrm{~cm}$, the mean age of the patients was $52.3 \pm 10.2$ years, and the serum ALT, albumin, and total bilirubin levels before the operation were $42.4 \pm 2.1 \mathrm{U} / \mathrm{L}, 32.2 \pm 3.7 \mathrm{~g} / \mathrm{L}$, and $29.6 \pm 7.6 \mu \mathrm{mol} / \mathrm{L}$, respectively. The mean duration of thermal ablation in this group was $14.0 \pm 4.3$ minutes. In the group without liver abscesses, the mean tumor diameter was $3.6 \pm 3.2 \mathrm{~cm}$ and the mean age was $54.8 \pm 11.3$ years. The mean serum ALT, albumin, and total bilirubin levels before the operation in this group were $36.5 \pm 4.6 \mathrm{U} / \mathrm{L}, 38.1 \pm 2.4 \mathrm{~g} / \mathrm{L}$, and $25.3 \pm$ $6.4 \mu \mathrm{mol} / \mathrm{L}$, respectively, and the mean duration of thermal ablation was $10.8 \pm 6.6$ minutes. Liver abscesses occurred in seven (4.0\%) of the 223 patients with child-pugh class B and child-pugh class C cirrhosis, in two (2.9\%) of the 68 that presented with alcoholic liver disease, in six (16.7\%) of the 36 that had porta hepatis tumors, and in six $(6.7 \%)$ of the 89 with biliary tract disease. Among the biliary tract disease patients, there were three with intrahepatic cholangiocarcinoma, one who underwent bilioenteric anastomosis, one with bacterial-infected cholelithiasis, and one who underwent sphincterotomy and endoscopic retrograde biliary drainage. Liver abscesses also developed in three $(42.8 \%)$ of the seven patients with intrahepatic cholangiocarcinoma, five (6.5\%) of the 77 with diabetes mellitus, six (2.1\%) of the 288 with multiple tumors, 11 (1.6\%) of the 655 with percutaneous punctures, one $1.4 \%$ ) of the 69 with local tumor progression, and four (1.3\%) of the 315 who underwent TACE 15 - 388 days before thermal ablation. The analysis of the potential risk factors showed that child-pugh class $\mathrm{B}$ or class $\mathrm{C}$ cirrhosis $(\mathrm{P}=0.0486)$, biliary tract disease $(\mathrm{P}=0.0305)$, diabetes mellitus $(\mathrm{P}=0.0344)$, and porta hepatis tumors $(\mathrm{P}=0.0123)$ were significant risk factors for liver abscess formation. Intrahepatic cholangiocarcinoma $(\mathrm{P}=0.0015)$ was observed to be an independent risk factor of liver abscess formation associated with biliary tract disease.

\section{Discussion}

Previous studies have reported that the incidence of liver abscess was approximately $0.3 \%-2.0 \%$ after $\operatorname{RFA}(20,21)$ 
Table 2. Baseline Characteristics of Patients With Liver Abscesses After Thermal Ablation

\begin{tabular}{|c|c|c|c|c|c|c|c|c|c|c|c|c|}
\hline Patient Sequence No. Basic Characteristics of Patients & 1 & 2 & 3 & 4 & 5 & 6 & 7 & 8 & 9 & 10 & 11 & 12 \\
\hline Gender & Male & Male & Male & Female & Male & Male & Male & Female & Male & Male & Male & Male \\
\hline Age, $y$ & 70 & 64 & 66 & 68 & 50 & 56 & 44 & 55 & 72 & 76 & 57 & 65 \\
\hline Maximum tumor diameter, $\mathrm{cm}$ & 4.5 & 2.2 & 4.6 & 3.2 & 2.4 & 5.5 & 2.4 & 2.3 & 3.0 & 2.8 & 3.5 & 8.5 \\
\hline Quantity of tumors & 1 & 3 & 2 & 1 & 1 & 1 & 3 & 1 & 1 & 2 & 2 & 2 \\
\hline Tumor position (segment) & IV & VIII & II,VII & IV & I & IV & VII,VIII & $\mathrm{v}$ & $\mathrm{VI}$ & $\mathrm{IV}, \mathrm{V}$ & $\mathrm{VI}$ & $\mathrm{II}, \mathrm{III}$ \\
\hline Number of abscesses & 1 & 1 & 2 & 1 & 1 & 1 & 1 & 1 & 1 & 2 & 1 & 2 \\
\hline Alcoholism history & Yes & No & No & No & No & Yes & No & No & No & No & No & No \\
\hline Liver cirrhosis history & Yes & No & Yes & Yes & No & No & Yes & No & Yes & Yes & No & Yes \\
\hline Biliary tract disease history & Yes & No & Yes & Yes & Yes & No & No & Yes & No & No & No & Yes \\
\hline Diabetes mellitus history & No & Yes & No & No & Yes & No & Yes & No & No & Yes & Yes & No \\
\hline First porta hepatis tumor & Yes & No & Yes & Yes & Yes & No & No & Yes & Yes & Yes & No & No \\
\hline Ever underwent TACE & Yes & No & No & No & No & Yes & No & No & Yes & No & No & Yes \\
\hline ALT, U/L & 33 & 21 & 46 & 77 & 35 & 35 & 42 & 55 & 36 & 48 & 19 & 88 \\
\hline Serum albumin, $\mathrm{g} / \mathrm{L}$ & 31 & 45 & 28 & 27 & 38 & 34 & 29 & 41 & 35 & 29 & 38 & 32 \\
\hline PT, \% & 57 & 77 & 60 & 44 & 90 & 85 & 48 & 76 & 58 & 42 & 100 & 55 \\
\hline Total bilirubin, $\mu \mathrm{mol} / \mathbf{L}$ & 33.5 & 11.0 & 37.4 & 44.4 & 31.0 & 14.5 & 19.3 & 66.2 & 28.0 & 36.5 & 24.2 & 33.0 \\
\hline Preoperative antibiotic application & Yes & No & No & No & Yes & Yes & No & Yes & Yes & Yes & Yes & No \\
\hline Postoperative antibiotic application & Yes & Yes & Yes & Yes & Yes & Yes & Yes & Yes & Yes & Yes & Yes & Yes \\
\hline Anesthesia method & Local & Int & Local & Int & Int & Loca & Local & Local & Int & Int & Int & Local \\
\hline Puncture method & Perc & Abd & Perc & Perc & Perc & Perc & Perc & Perc & Perc & Perc & Perc & Perc \\
\hline Thermal ablation process & RFA & MWA & MWA & RFA & RFA & RFA & MWA & RFA & RFA & MWA & RFA & MWA \\
\hline Thermal ablation time, min & 36 & 11 & 29 & 20 & 32 & 40 & 9 & 14 & 21 & 22 & 58 & 65 \\
\hline Forming time, $d$ & 55 & 37 & 3 & 32 & 5 & 3 & 28 & 12 & 6 & 20 & 4 & 1 \\
\hline Diagnostic method for abscess & CT & CT & CT & CT & MR & ст & CT & СT & Ст & MR & MR & CT \\
\hline Outcome & Rec & Rec & Rec & Death & Rec & Rec & $\operatorname{Rec}$ & Rec & Rec & $\operatorname{Rec}$ & $\operatorname{Rec}$ & Rec \\
\hline
\end{tabular}

Abbreviations: Abd, Abdominal; Asp, Aspiration; Dra, Drainage; HCC, hepatocellular carcinoma; ICC, intrahepatic cholangiocarcinoma; Int, intravenous; MHC, metastatic hepatic carcinoma; Neg, negative; Ope, Operation; Perc, Percuta neous; Positive, Positive; Rec, Recovery.

Figure 1. A 66-Year-Old Patient With Multiple Tumors Who Underwent RFA Via Percutaneous Puncture
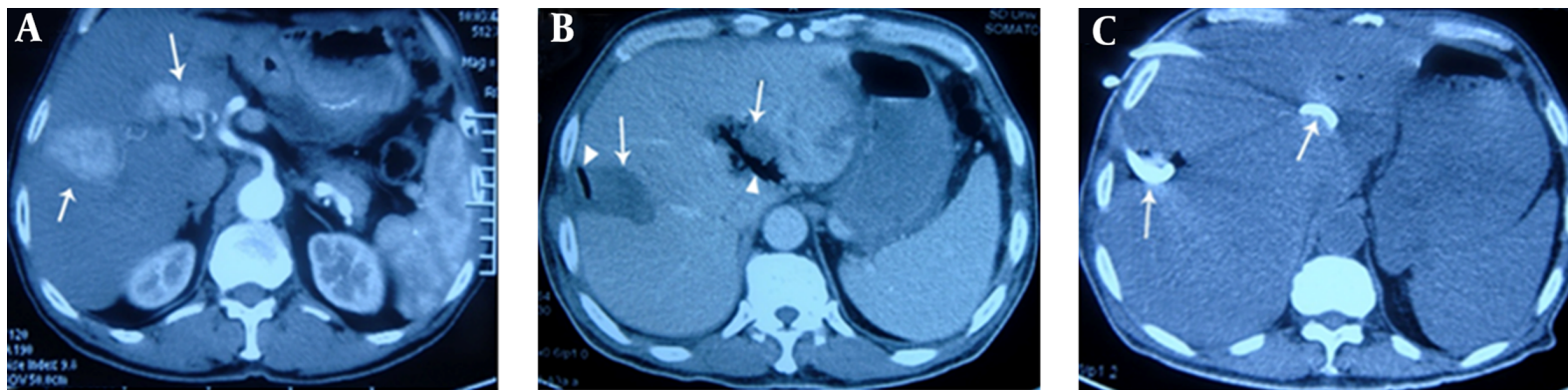

A, contrast-enhanced transverse CT obtained during the arterial phase shows irregular liver tumors (arrows) with maximum diameters of $4.6 \mathrm{~cm}$ and $3.5 \mathrm{~cm}$, in liver segments VII and II, respectively. The latter tumor is located in the porta hepatis; B, on the 6th postoperative day, the patient underwent CT due to fever and chills. CT showed gas (triangular mark) forming in the low-attenuation ablation zone (arrow), indicating formation of a liver abscess. Cultures from puncture aspiration were positive for Escherichia coli; $\mathrm{C}$, in addition to treatment with carbapenem antibiotics, catheter drainage (arrows) by percutaneous puncture under ultrasonic guidance was administered, and pus was aspirated. Four weeks later, the patient had recovered. 

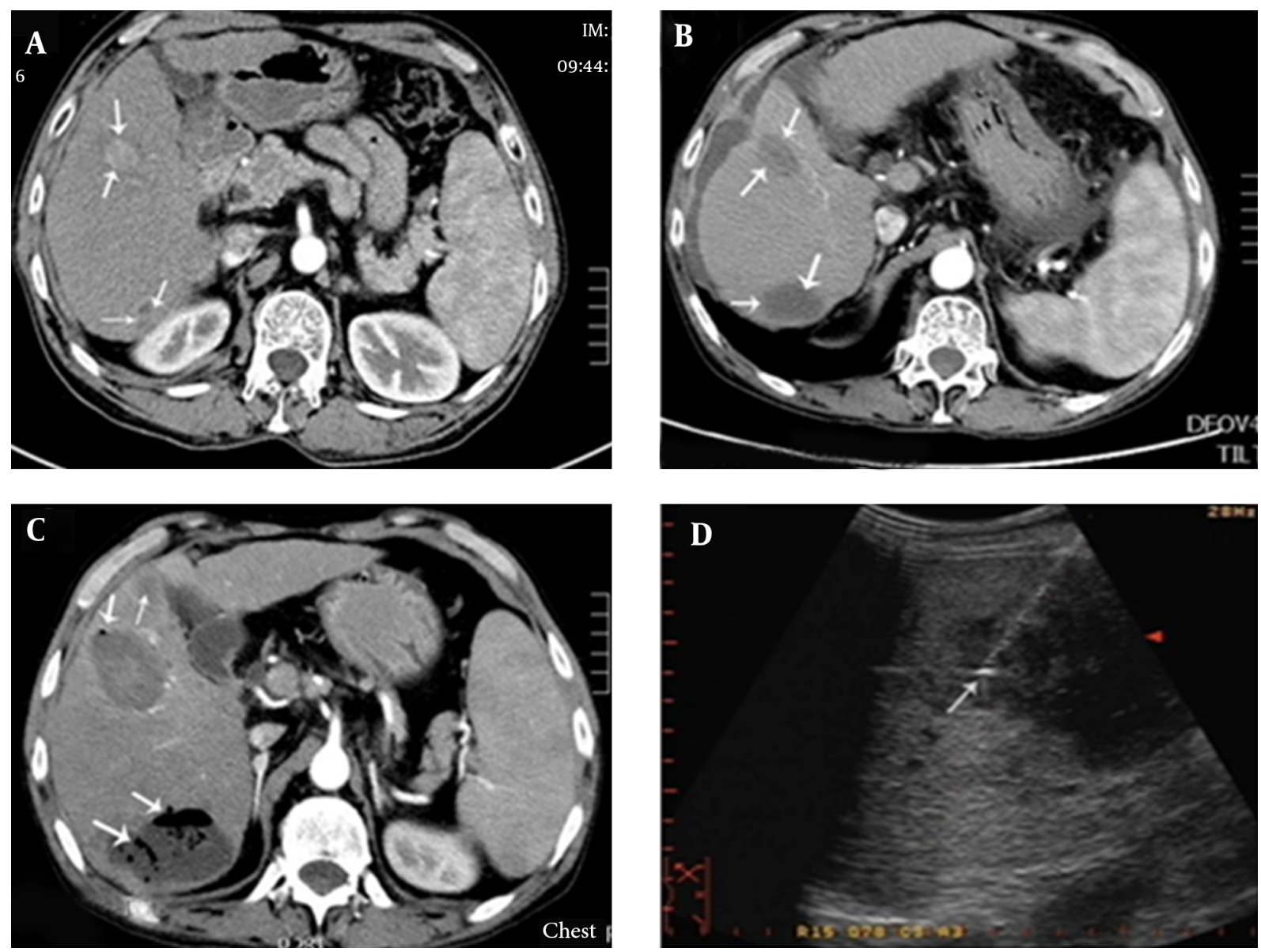

A, contrast-enhanced transverse CT imaging shows a $2.8 \times 2.1 \mathrm{~cm}$ liver tumor (arrow) in segment IV and an irregular tumor (arrow) measuring approximately $2 \times 1.5 \mathrm{~cm}$ located close to the top of the right kidney in segment VI; B, after MWA, contrast-enhanced transverse CT imaging showed an oval low-density lesion (arrows); C, three days after the operation, hyperpyrexia occurred, with body temperature reaching $39.0^{\circ} \mathrm{C}$. One week after the operation, contrast-enhanced $\mathrm{CT}$ showed an expanded ablation zone with pneumatosis, suggesting the formation of a liver abscess (arrows); D, catheter drainage of the liver abscess.

Table 3. Influence of Treatment Method on Development of Liver Abscess After Thermal Ablation

\begin{tabular}{|c|c|c|c|c|}
\hline & RFA & MWA & Ablation Combined With PEI & Ablation Alone \\
\hline Number of patients with liver abscesses & 7 & 5 & 0 & 12 \\
\hline Number of patients without liver abscesses & 378 & 299 & 73 & 606 \\
\hline Total & 385 & 306 & 73 & 618 \\
\hline$\chi^{2}$ value & 1.18 & 1.18 & 15.74 & 15.74 \\
\hline
\end{tabular}

RFA and MWA in the incidence of liver abscess. Knowledge pertaining to the risk factors for liver abscess formation after thermal ablation is likely to be valuable in reducing the occurrence of this complication, shortening hospitalization periods, and reducing the associated mortality rate.
Similar to the syndrome that occurs after TACE, fever can occur after thermal ablation (25). Generally, body temperature reaches a peak three days after the procedure and then gradually declines. If the fever lasts longer than two weeks, or the hyperpyrexia (body temperature of $>38.5^{\circ} \mathrm{C}$ ) 
Table 4. Relationships Between Risk Factor Variables and Abscess Formation After RFA and MWA Treatment ${ }^{\mathrm{a}}$

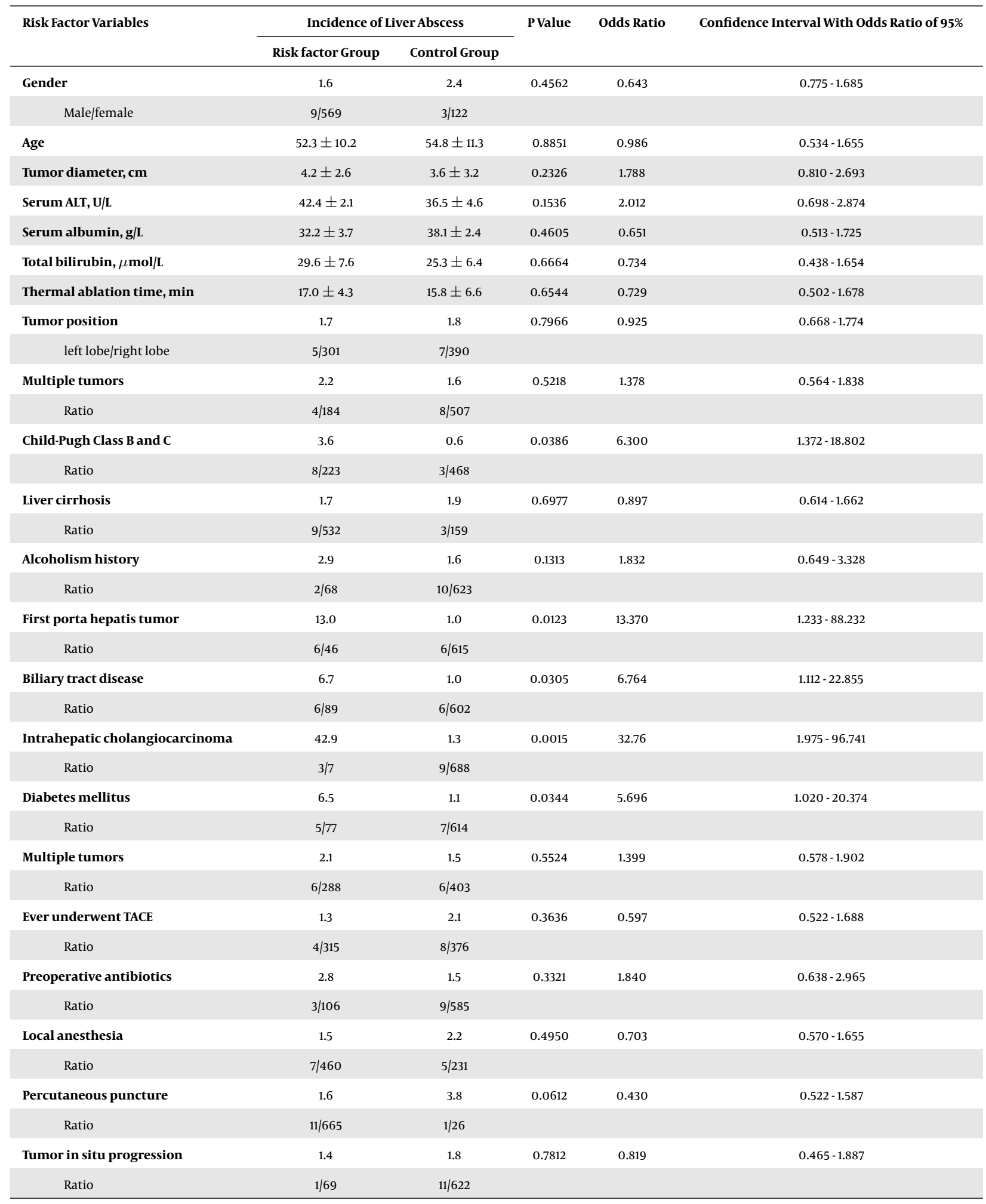

${ }^{\mathrm{a}}$ Values are expressed as mean $\pm \mathrm{SD}$ or $\%$. 
lasts longer than three days, the possibility of liver abscess formation should be considered (26). The mechanism by which patients develop a liver abscess after thermal ablation is not well understood. The most common routes of infection resulting in bacterial liver abscesses include the bile ducts, portal vein, hepatic artery, and direction invasion from other infected cavities, especially the biliary tract (27). Operations on the biliary tract, such as bilioenteric anastomosis, may damage the integrity of the extrahepatic or intrahepatic bile duct epithelium and allow retrograde passage of bacteria from the intestinal tract into the liver, resulting in proliferation in the ablation region. de Baere et al. (28) demonstrated that liver abscesses developed in seven out of 582 patients following thermal ablation. All three patients who had undergone bilioenteric anastomosis developed liver abscesses. This is likely due to injury to the biliary tract, allowing intestinal bacteria to contaminate the necrosis zone after ablation (29). In our study, the incidence of liver abscess was as high as $6.7 \%$, which indicated that biliary duct disease is related to liver abscess formation after thermal ablation. We suspect that along with bilioenteric anastomosis, the presence of bile duct stones, biliary tract stent implantation, intrahepatic cholangiocarcinoma, and other bile duct diseases, such as biliary abnormalities, may be important risk factors (23).

Intrahepatic cholangiocarcinoma usually causes obstruction of the biliary tract because of inadequate drainage of bile, which increases the probability of liver abscess due to retrograde infection. Kim et al. (30) reported that liver abscesses occurred in only one (5.0\%) of 20 intrahepatic cholangiocarcinoma patients undergoing hepatectomy after ablation. In the current study, three $(42.8 \%)$ of seven patients with intrahepatic cholangiocarcinoma developed abscesses after ablation procedures because of increased risk due to ascending biliary infection.

Another mechanism of liver abscess formation is biloma followed by concurrent infection $(31,32)$. When the tumor is located in the porta hepatis or the distance between the tumor and the main bile duct is $<1 \mathrm{~cm}$, injury to the main bile duct can be inevitable because it is necessary to create a $0.5-1 \mathrm{~cm}$ ablation boundary beyond the range of the tumor. This can result in bilomas connected to the main bile duct in the ablation zone, and liver abscesses may occur due to retrograde infection. This may explain why abscesses occurred in $16.7 \%$ of patients who had porta hepatis tumors in the present study. Some reports have stated that RFA and MWA treatments should not be applied to tumors in the center of the porta hepatis (33).

After analyzing the 77 patients with diabetes mellitus in this study, we found that diabetes might have been related to the development of abscesses in five of them. A study by Thomsen et al. on a large cohort in Denmark showed a high incidence of liver abscesses after RFA for hepatic malignancy in patients with diabetes mellitus (34). Liang et al. analyzed complications associated with 1,136 patients after MWA, and found that out of five patients with liver abscesses, two had underlying diabetes mellitus (40\%) (10). Hyperglycemia enhances bacterial growth, inhibits the cellular immune response, and weakens the bactericidal strength of the hematological system, possibly facilitating the ability of bacteria to cause infection, to reproduce, and to form liver abscesses.

A study by Choi et al. showed that child-pugh class B cirrhosis was not a risk factor for liver abscess formation after RFA (22). In contrast, the current analysis demonstrated that patients with child-pugh class B or class $C$ cirrhosis had a higher rate of liver abscesses than patients at the class A stage, and one patient with class C cirrhosis died of septicemia. Although active measures were adopted, there were two cases of liver abscesses, with the incidence reaching $11.7 \%$. This might be one of the reasons the current results differed from previous reports (22). In Europe, patients with child-pugh class $\mathrm{C}$ cirrhosis are recommended to receive only palliative support, including pain, nutritional, and psychological management (35). However, in China, many patients with child-pugh class $\mathrm{C}$ cirrhosis choose thermal ablation even after receiving detailed information about its risks versus benefits, as there is no other effective therapy except for liver transplantation, which is expensive. In addition, donor livers are frequently of limited availability.

Apart from mitigating the risk factors highlighted above, it has been suggested that treatment combining thermal ablation with PEI could reduce the incidence of liver abscesses due to micro-bile duct sclerosis caused by anhydrous alcohol (20). This may inhibit migration and infection by bacteria. Additionally, PEI in tumors close to the bile duct might increase the therapeutic efficacy while decreasing thermal injury to the bile duct (35), thus reducing the incidence of liver abscesses.

Although it is debatable whether antibiotics should be given to all patients who receive thermal ablations, most reports suggest that those with the highest risk of infection should be given prophylactic antibiotics $(30,31)$. Hoffmann et al. applied broad-spectrum antibiotic treatment to patients undergoing bilioenteric anastomosis operations, and liver abscesses developed in only a single patient who underwent TACE (36). In our study, liver abscesses occurred in two (3\%) out of 66 patients with biliary tract disease who had received prophylactic antibiotics, and in four (17.3\%) out of 23 patients who did not receive prophylactic antibiotics. This suggests that the use of prophylactic antibiotics is necessary for patients with a high risk of infec- 
tion, especially those undergoing biliary tract procedures. Further prospective studies are required to determine the effectiveness of aggressive antibiotic use.

Our study had some limitations. First, the data were derived from two hospitals, with different environments, equipment, and doctor expertise. Second, we did not conduct a more detailed analysis according to tumor size. For example, the incidence of liver abscesses in patients with varying tumor diameters may be different. Finally, because this study was a retrospective analysis, the frequency of thermal ablations could not be controlled, and there may have been selection bias.

In conclusion, after thermal ablation of liver cancer, the overall incidence of liver abscesses was relatively low, and there was no significant difference in the rate of abscesses between the RFA and MWA treatments. Our results suggest that operators should pay more attention to the risk factors for abscess formation in patients with biliary tract disease, child-pugh class $B$ and class $C$ cirrhosis, diabetes, and tumors in the porta hepatis. For high-risk patients, we recommend following the appropriate indications for thermal ablation.

\section{Footnote}

Authors' Contribution: Xiu-Feng Su and Na Li contributed equally to this work and should be considered co-first authors. Xiu-Feng Su and Na Li collected and analyzed the data, and drafted the manuscript; Xu-Fang Chen and Lei Zhang provided analytical oversight, and Ming Yan designed and supervised the study and revised the manuscript. All authors have read and approved the final version to be published.

\section{References}

1. Shiina S, Tateishi R, Arano T, Uchino K, Enooku K, Nakagawa H, et al. Radiofrequency ablation for hepatocellular carcinoma: 10-year outcome and prognostic factors. Am J Gastroenterol. 2012;107(4):569-77. doi: 10.1038/ajg.2011.425. [PubMed: 22158026] quiz 578.

2. Xu G, Qi FZ, Zhang JH, Cheng GF, Cai Y, Miao Y. Meta-analysis of surgical resection and radiofrequency ablation for early hepatocellular carcinoma. World JSurg Oncol. 2012;10:163. doi:10.1186/1477-7819-10-163. [PubMed: 22897815].

3. Fu C, Liu N, Deng Q, Li X, Ma K, Bie P. Radiofrequency ablation vs. surgical resection on the treatment of patients with small hepatocellular carcinoma: a system review and meta-analysis of five randomized controlled trials. Hepatogastroenterology. 2014;61(134):17229. [PubMed: 25436369].

4. Feng Q, Chi Y, Liu Y, Zhang L, Liu Q. Efficacy and safety of percutaneous radiofrequency ablation versus surgical resection for small hepatocellular carcinoma: a meta-analysis of 23 studies. J Cancer Res Clin Oncol. 2015;141(1):1-9. doi: 10.1007/s00432-014-1708-1. [PubMed: 24889505].
5. Groeschl RT, Pilgrim CH, Hanna EM, Simo KA, Swan RZ, Sindram D, et al. Microwave ablation for hepatic malignancies: a multiinstitutional analysis. Ann Surg. 2014;259(6):1195-200. doi: 10.1097/SLA.0000000000000234. [PubMed: 24096760].

6. Clinical Practice Guidelines for Hepatocellular Carcinoma. . Clinical Practice Guidelines for Hepatocellular Carcinoma - The Japan Society of Hepatology 2009 update. Hepatol Res. 2010;40 Suppl 1:2-144. doi: 10.1111/j.1872-034X.2010.00650.x. [PubMed: 20586808].

7. Takaki H, Yamakado K, Nakatsuka A, Yamada T, Shiraki K, Takei Y, et al. Frequency of and risk factors for complications after liver radiofrequency ablation under CT fluoroscopic guidance in 1500 sessions: single-center experience. AJR Am J Roentgenol. 2013;200(3):65864. doi: 10.2214/AJR.12.8691. [PubMed: 23436859].

8. Bertot LC, Sato M, Tateishi R, Yoshida H, Koike K. Mortality and complication rates of percutaneous ablative techniques for the treatment of liver tumors: a systematic review. Eur Radiol. 2011;21(12):2584-96. doi: 10.1007/s00330-011-2222-3. [PubMed: 21858539].

9. Ding J, Jing X, Liu J, Wang Y, Wang F, Wang Y, et al. Complications of thermal ablation of hepatic tumours: comparison of radiofrequency and microwave ablative techniques. Clin Radiol. 2013;68(6):608-15. doi: 10.1016/j.crad.2012.12.008. [PubMed: 23399463].

10. Liang P, Wang Y, Yu X, Dong B. Malignant liver tumors: treatment with percutaneous microwave ablation-complications among cohort of 1136 patients. Radiology. 2009;251(3):933-40. doi: 10.1148/radiol.2513081740. [PubMed:19304921].

11. Livraghi T, Meloni F, Solbiati L, Zanus G, Collaborative Italian Group using AS. Complications of microwave ablation for liver tumors: results of a multicenter study. Cardiovasc Intervent $\mathrm{Ra}$ diol. 2012;35(4):868-74. doi: 10.1007/s00270-011-0241-8. [PubMed: 21833809].

12. Ye SL QS, Wu MC. Expert consensus of the Standardized diagnosis and treatment for Hepatocellular carcinoma. J Clin Hepatol. 2009;12(5).

13. Zhang X, Zhou L, Chen B, Hu S, Wachtel MS, Frezza EE. Microwave ablation with cooled-tip electrode for liver cancer: an analysis of 160 cases. Minim Invasive Ther Allied Technol. 2008;17(5):303-7. doi: 10.1080/13645700802383926. [PubMed: 18942003].

14. Tanaka S, Shimada M, Shirabe K, Taketomi A, Maehara S, Tsujita E, et al. Surgical radiofrequency ablation for treatment of hepatocellular carcinoma: an endoscopic or open approach. Hepatogastroenterology. 2009;56(93):1169-73. [PubMed: 19760963].

15. Salama IA, Korayem E, ElAbd O, El-Refaie A. Laparoscopic ultrasound with radiofrequency ablation of hepatic tumors in cirrhotic patients. J Laparoendosc Adv Surg Tech A. 2010;20(1):39-46. doi: 10.1089/lap.2009.0208. [PubMed: 20100059].

16. Zhang XG, Zhang ZL, Hu SY, Wang YL. Ultrasound-guided ablative therapy for hepatic malignancies : a comparison of the therapeutic effects of microwave and radiofrequency ablation. Acta Chir Belg. 2014;114(1):40-5. [PubMed: 24720137].

17. Martin RC, Scoggins CR, McMasters KM. Safety and efficacy of microwave ablation of hepatic tumors: a prospective review of a 5-year experience. Ann Surg Oncol. 2010;17(1):171-8. doi: 10.1245/s10434-0090686-z. [PubMed: 19707829].

18. Rhim H, Lim HK, Kim YS, Choi D. Percutaneous radiofrequency ablation with artificial ascites for hepatocellular carcinoma in the hepatic dome: initial experience. AJR Am J Roentgenol. 2008;190(1):91-8. doi: 10.2214/AJR.07.2384. [PubMed: 18094298].

19. Song I, Rhim H, Lim HK, Kim YS, Choi D. Percutaneous radiofrequency ablation of hepatocellular carcinoma abutting the diaphragm and gastrointestinal tracts with the use of artificial ascites: safety and technical efficacy in 143 patients. Eur Radiol. 2009;19(11):2630-40. doi: 10.1007/s00330-009-1463-x. [PubMed: 19557416].

20. Kurokohchi K, Watanabe S, Yoneyama H, Deguchi A, Masaki T, Himoto $\mathrm{T}$, et al. A combination therapy of ethanol injection and radiofrequency ablation under general anesthesia for the treatment of hepatocellular carcinoma. World J Gastroenterol. 2008;14(13):2037-43. [PubMed: 18395903]. 
21. Shibata T, Yamamoto Y, Yamamoto N, Maetani Y, Shibata T, Ikai I, et al. Cholangitis and liver abscess after percutaneous ablation therapy for liver tumors: incidence and risk factors. J Vasc Interv Radiol. 2003;14(12):1535-42. [PubMed: 14654488].

22. Choi D, Lim HK, Kim MJ, Kim SJ, Kim SH, Lee WJ, et al. Liver abscess after percutaneous radiofrequency ablation for hepatocellular carcinomas: frequency and risk factors. AJR Am J Roentgenol. 2005;184(6):1860-7. doi: 10.2214/ajr.184.6.01841860. [PubMed: 15908543].

23. Ahmed M, Solbiati L, Brace CL, Breen DJ, Callstrom MR, Charboneau JW, et al. Image-guided tumor ablation: standardization of terminology and reporting criteria-a 10-year update. Radiology. 2014;273(1):241-60. doi: 10.1148/radiol.14132958. [PubMed: 24927329].

24. Wang XH, Yu J, Liang P, Yu XL, Cheng ZG, Han ZY, et al. [Percutaneous cooled-tip microwave ablation under ultrasound guidance for primary liver cancer: analysis of major complications in 693 patients]. Zhonghua Zhong Liu Za Zhi. 2012;34(12):945-9. doi: 10.3760/cma.j.issn.0253-3766.2012.12.014. [PubMed: 23336384].

25. Oshima S, Tani N, Takaishi K, Hirano M, Makari Y, Hoshi M, et al. [Clinical evaluation of the risk factors for liver abscess after TACE or RFA]. Gan To Kagaku Ryoho. 2014;41(12):2113-5. [PubMed: 25731440].

26. Rhim H, Yoon KH, Lee JM, Cho Y, Cho JS, Kim SH, et al. Major complications after radio-frequency thermal ablation of hepatic tumors: spectrum of imaging findings. Radiographics. 2003;23(1):123-34. doi: 10.1148/rg.231025054. [PubMed: 12533647] discussion 134-6.

27. Hope WW, Vrochides DV, Newcomb WL, Mayo-Smith WW, Iannitti DA. Optimal treatment of hepatic abscess. Am Surg. 2008;74(2):178-82. [PubMed: 18306874].

28. de Baere T, Risse O, Kuoch V, Dromain C, Sengel C, Smayra T, et al. Adverse events during radiofrequency treatment of 582 hepatic tumors. AJRAm J Roentgenol. 2003;181(3):695-700. doi:10.2214/ajr.181.3.1810695. [PubMed: 12933462].

29. Giorgio A, Ferraioli G. Liver abscess formation after radiofrequency ablation in patients with hepatocellular carcinoma. AJR Am J Roentgenol. 2006;186(2):582. doi: 10.2214/AJR.06.5011. [PubMed: 16423976] author reply 582-3.

30. Kim JH, Won HJ, Shin YM, Kim PN, Lee SG, Hwang S. Radiofrequency ablation for recurrent intrahepatic cholangiocarcinoma after curative resection. Eur J Radiol. 2011;80(3):e221-5. doi: 10.1016/j.ejrad.2010.09.019. [PubMed: 20950977].

31. Kondo Y, Shiina S, Tateishi R, Arano T, Uchino K, Enooku K, et al. Intrahepatic bile duct dilatation after percutaneous radiofrequency ablation for hepatocellular carcinoma: impact on patient's prog nosis. Liver Int. 2011;31(2):197-205. doi: 10.1111/j.1478-3231.2010.02415.x. [PubMed: 21159122].

32. Chang IS, Rhim H, Kim SH, Kim YS, Choi D, Park Y, et al. Biloma formation after radiofrequency ablation of hepatocellular carcinoma: incidence, imaging features, and clinical significance. AJRAm J Roentgenol. 2010;195(5):1131-6. doi: 10.2214/AJR.09.3946. [PubMed: 20966318].

33. Minami Y, Kudo M. Radiofrequency ablation of hepatocellular carcinoma: a literature review. Int J Hepatol. 2011;2011:104685. doi: 10.4061/2011/104685. [PubMed: 21994847].

34. Keynan Y, Rubinstein E. Diabetes mellitus and pyogenic liver abscess: risk and prognosis. Clin Infect Dis. 2007;45(6):801. doi:10.1086/521170. [PubMed: 17712769].

35. Azab M, Zaki S, El-Shetey AG, Abdel-Moty MF, Alnoomani NM, Gomaa AA, et al. Radiofrequency ablation combined with percutaneous ethanol injection in patients with hepatocellular carcinoma. Arab J Gastroenterol. 2011;12(3):113-8. doi: 10.1016/j.ajg.2011.07.005. [PubMed: 22055587].

36. Hoffmann R, Rempp H, Schmidt D, Pereira PL, Claussen CD, Clasen S Prolonged antibiotic prophylaxis in patients with bilioenteric anastomosis undergoing percutaneous radiofrequency ablation. JVasc Interv Radiol. 2012;23(4):545-51. doi: 10.1016/j.jvir.2011.12.025. [PubMed: 22365593]. 